\title{
Colonic Perforation Associated with Necrotizing Fasciitis in a Patient Receiving Tyrosine Kinase Inhibitor (Pazopanib) for Recurrent Retroperitoneal Renal Cell Carcinoma
}

\author{
Arda Akoluk ${ }^{\mathrm{a}}$, Steven Douedi ${ }^{\mathrm{a}, \mathrm{g}}$, Jaraad Dattadeen ${ }^{\mathrm{a}}$, Victoria Grille ${ }^{\mathrm{b}}$, \\ Esther Kaufman ${ }^{\mathrm{c}}$, Edward Liud, Glenn Parker ${ }^{\mathrm{b}}$, e ${ }^{\mathrm{d}}$ Kenneth Nahum ${ }^{\mathrm{f}}$
}

\begin{abstract}
Pazopanib, under the trade name of votrient, is a potent tyrosine-kinase growth factor receptor inhibitor used in the treatment of latestage kidney cancer and soft tissue sarcoma. Rarely this drug has been associated with gastrointestinal perforations; however, prior studies have not shown an association with necrotizing fasciitis. We present a case of suspected pazopanib-induced extensive colonic perforation resulting in a severe necrotizing fasciitis. As this is a potentially rare complication of this targeted therapy, our goal is to heighten the awareness of colonic perforation as it is only seen in $0.9 \%$ of patients on pazopanib. The staged operative management and multidisciplinary approach throughout this patient's care allowed for complete recovery from a life-threatening diagnosis.
\end{abstract}

Keywords: Necrotizing fasciitis; Pazopanib; Colon; Perforation; Gastrointestinal; Kidney; Cancer

\section{Introduction}

Necrotizing fasciitis (NF) is a rare and rapidly life-threatening,

\footnotetext{
Manuscript submitted July 8, 2020, accepted July 28, 2020

Published online August 22, 2020

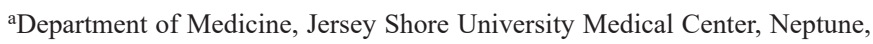
NJ 07753, USA

${ }^{b}$ Department of Surgery, Jersey Shore University Medical Center, Neptune, NJ 07753, USA

'Department of Pediatrics, Jersey Shore University Medical Center, Neptune, NJ 07753, USA

dDepartment of Infectious Disease, Jersey Shore University Medical Center, Neptune, NJ 07753, USA

${ }^{\mathrm{e} H a c k e n s a c k}$ Meridian School of Medicine at Seton Hall University Department of Surgery, Jersey Shore University Medical Center, Neptune, NJ 07753, USA fDepartment of Oncology, Jersey Shore University Medical Center, Neptune, NJ 07753, USA

gCorresponding Author: Steven Douedi, Department of Surgery, Jersey Shore University Medical Center, Neptune, NJ 07753, USA.

Email: Steven.Douedi@hackensackmeridian.org
}

doi: https://doi.org/10.14740/jcs416 gangrenous soft-tissue infection usually caused by toxin-producing bacteria that typically presents with fevers, severe pain, tenderness, erythema, and systemic toxicity [1]. Usual risk factors include any form of penetrating trauma, breach of the skin or mucosa, either by major traumatic or minor (surgical procedures, lacerations, abrasions) causes, and pregnancy or childbirth, which then lead to infection and necrosis of soft tissue [2]. In this case, the drug pazopanib was used for the patient's renal cell carcinoma (RCC). Major adverse effects of pazopanib include gastrointestinal perforation, hand-foot skin reaction, heart failure, hemorrhage, hepatoxicity and hypertension [3]. The drug may have induced a gastrointestinal perforation or break in the mucosa, which ultimately leads to a retroperitoneal colonic perforation. The retroperitoneal perforation led to development of NF as it penetrated the posterior musculature of the flank/back. This clinical scenario is a rare presentation and was successfully managed to a successful recovery.

\section{Case Report}

A 50-year-old woman had a history of RCC, status post right nephrectomy in 2015 , followed by a right lower lobectomy (RLL) in 2018 for an isolated lung metastasis. The tyrosine kinase inhibitor, pazopanib, was given following the RCC for targeted therapy. She presented to the emergency department with 3 days of progressively worsening right flank pain without any associated fevers, chills, abdominal pain, or changes in bowel or urinary habits. On physical exam her temperature was 101.8 degrees Fahrenheit, blood pressure was 107/72 mm $\mathrm{Hg}$, and heart rate was 111 beats per minute (bpm). She was non-toxic appearing and had a $4-\mathrm{cm}$ raised erythematous region on her right flank/back with crepitus present. Remainder of physical exam was unremarkable. Initial abdominal and pelvic computed tomography (CT) scan with contrast showed a complex fluid collection with air in the right renal fossa extending into the subcutaneous tissues of the back (Fig. 1). Patient subsequently underwent a gastrograffin enema, which showed a colonic perforation with contrast extravasation from the hepatic flexure (Fig. 2). She was immediately taken to the operating room in which a large $20 \times 11 \mathrm{~cm}$ submuscular cavity was encountered, extending to paraspinal midline and a deeper cavity of approximately $6 \times 8 \mathrm{~cm}$ containing a loop of 


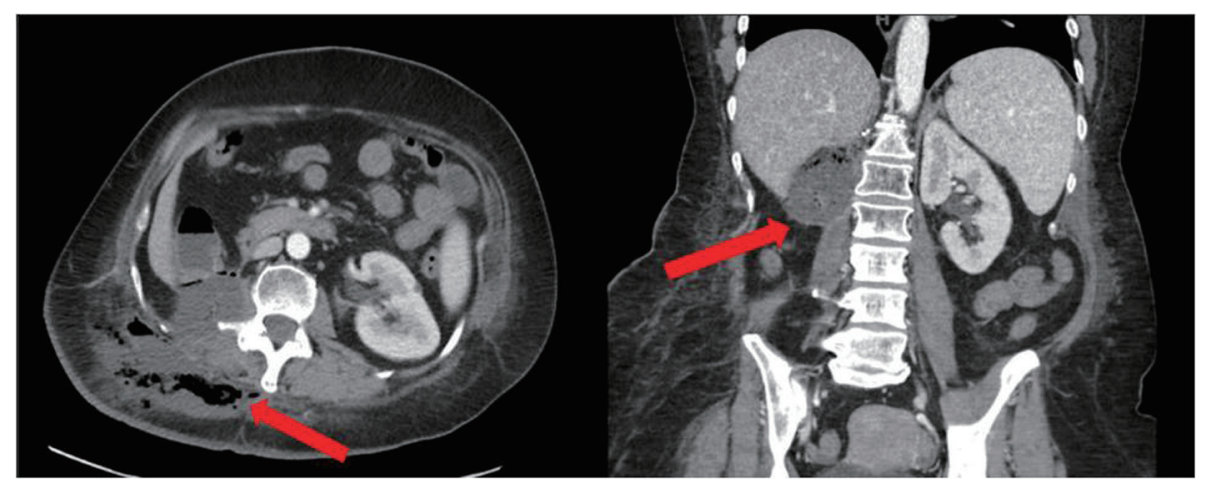

Figure 1. CT of the abdomen and pelvis with contrast showing a complex fluid collection with air in the right renal fossa (red arrows) extending into the subcutaneous tissues of the back. CT: computed tomography.

bowel with two perforations, feeding stool into both cavities. After initial source control with drainage and debridement of the posterior infected tissues, she was optimized and prepared for definitive surgery. The patient subsequently underwent a right hemicolectomy with primary ileo-transverse colostomy, repair of right paramedian incisional hernia (from prior right nephrectomy) along with left rectus abdominus flap to close the opening in her back in the renal fossa (Figs. 3, 4). Pathology of the resected specimen showed suppurative inflammation and extensive necrosis consistent with necrotizing fasciitis, without mucosal abnormalities, diverticulitis, or malignant cells (Fig. 5). A biologic mesh was then attached to the latissimus dorsi muscle for closure and additional coverage. The patient had a complete recovery with delayed primary closure of her back wound over 19 days and subsequently discharged.

\section{Discussion}

NF is a destructive gangrenous soft-tissue infection that typically presents with fever, chills, extreme tenderness, and ery-

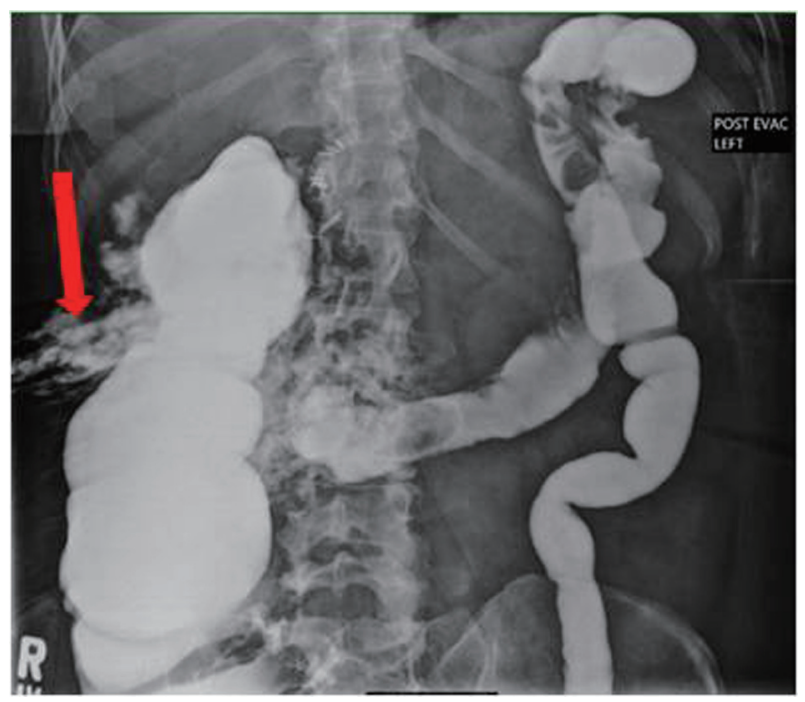

Figure 2. Gastrograffin enema showing colonic perforation with contrast extravasation (arrow) from the hepatic flexure. thema [1]. NF is a relatively rare disease with an annual incidence of 1,000 cases in the USA, and global prevalence of 0.040 cases per 1,000 person-years [4]. This number has been increasing since 1980 for reasons that are yet to be determined [5]. It has a predilection towards men versus women by a ratio of 3:1, most possibly due to incidence of Fournier's gangrene in men [6].

There are two different types of NF and the organisms that cause it to distinguish them from each other. Type 1, the more complex of the two, is caused by a mixture of both aerobe and anaerobe species and is more commonly seen in patients who are immunocompromised or have chronic diseases such as diabetes [7]. It usually presents in conditions that are favorable

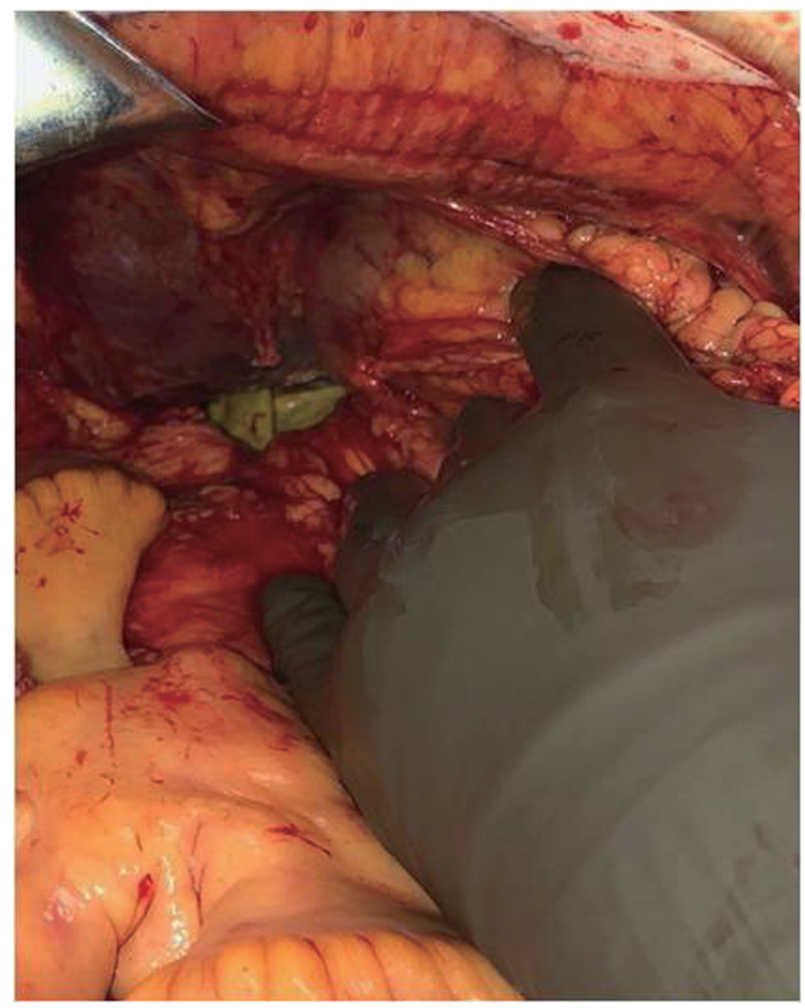

Figure 3. Penrose drain attached to left rectus abdominus flap for coverage of right renal fossa. 


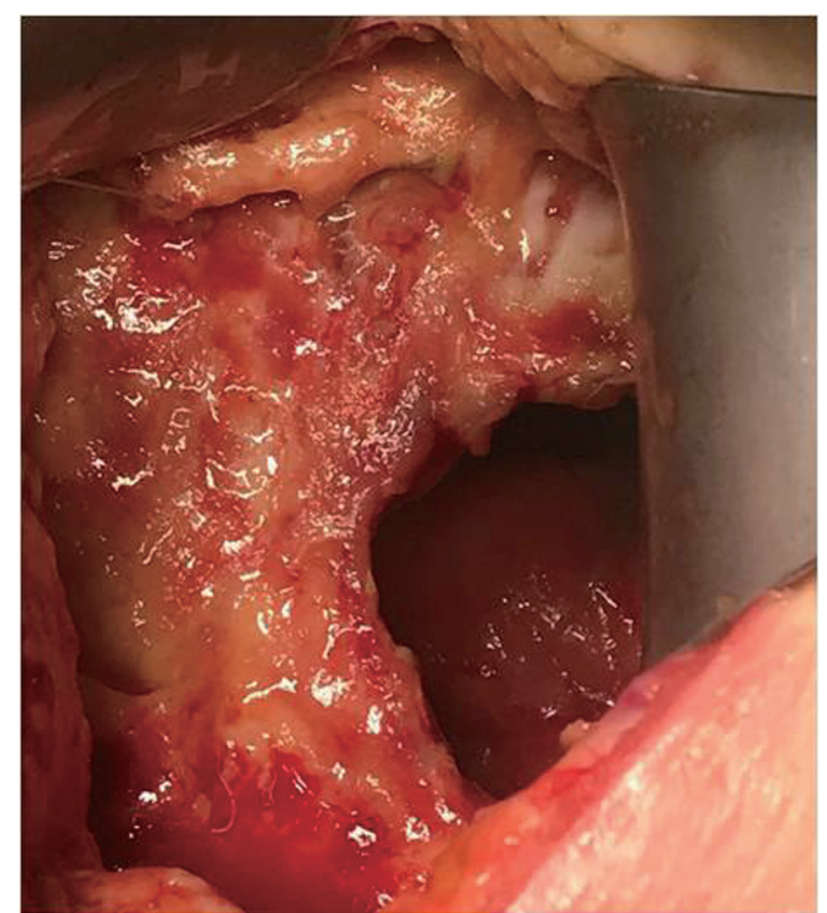

Figure 4. Posterior view from back with intraluminal view of ascending colon.

for polymicrobial growth including diabetics, fistulas, rectal fissures, hemorrhoids, surgeries and episiotomies [8]. Type 1 is classically associated with crepitus and suggests gas-forming organisms but is not always present [1]. Type 2, unlike type 1 , can occur in any age group and even in those without prior illness. It is monomicrobial in nature, with group A streptococcus being the most common, followed by methicillin-resistant Staphylococcus aureus (MRSA) [7]. Less common pathogens such as Vibrio vulnificus, Clostridium spp, and Aeromonus hydrophila may also represent a third type [9].

Vascular endothelial growth factor (VEGF) is an important and predominant factor promoting angiogenesis [10]. Development and growth of tumor cells, as well as formation of metastatic disease, relies on angiogenesis. Pazopanib, a VGEF-tyrosine kinase inhibitor, works well to decrease endothelial cell proliferation and angiogenesis, making it suitable in the treatment of metastatic lung cancer [11]. In this case, the patient had been on pazopanib for 24 months. This treatment is not without side effects, which include hypertension, proteinuria, and fatigue [11]. Additional complications include thromboembolism, bleeding, neurological complications, myelosuppression and, most relevant to this case, bowel perforation [11]. In randomized clinical control trials, gastrointestinal perforation or fistula was reported in $0.9 \%(5 / 586)$ of patients receiving votrient [12]. Fatal perforation events occurred in $0.3 \%(2 / 586)$ of these patients [12]. It is to be used with caution in patients at risk for these events and they should be monitored for signs and symptoms. Specifically, colonic perforation has been reported in $0.9 \%$ of patients treated with pazopanib $[13,14]$. However, NF being linked to complications of pazopanib has not been reported.

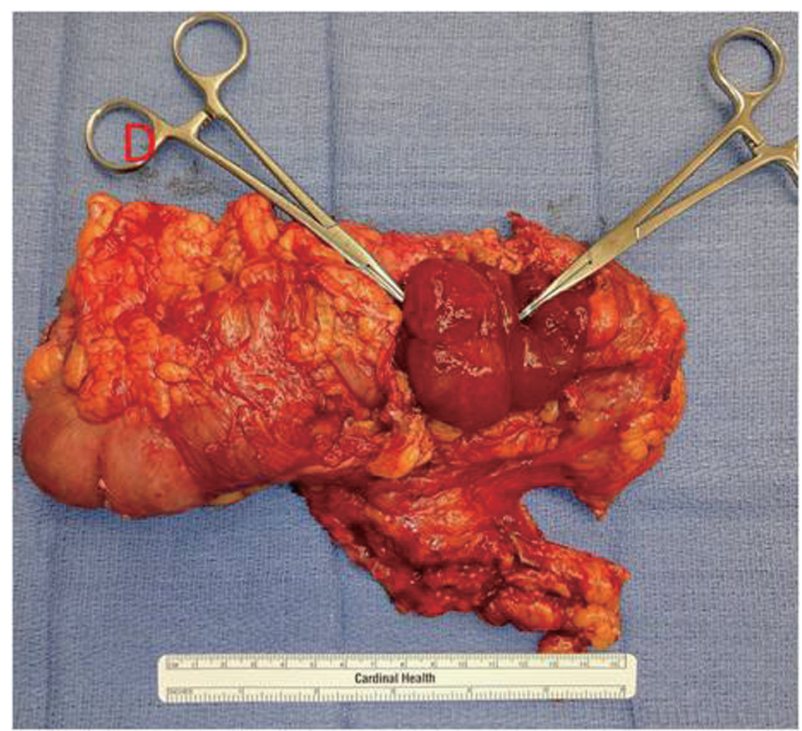

Figure 5. Giant colonic fistula with hemostats proximal and distal. Pathology of resected colon: suppurative inflammation and extensive necrosis consistent with NF, no mucosal abnormalities, diverticulitis, or malignancy. NF: necrotizing fasciitis.

Gastrointestinal perforation typically presents with an acute abdomen and diagnosis is usually obvious. However, the unusual presentation without overt peritonitis and sepsis can be attributed to the perforation being located in the retroperitoneal space where the right kidney had been removed, along with the use of narcotics for chronic pain. Additionally, NF can present differently in immunocompromised patients (active malignancy) as opposed to immune-competent patients as seen in the study by Keung et al [15]. Diagnosing these types of patients can pose a challenge. Wong et al developed a laboratory evaluation known as the Laboratory Risk Indicator for Necrotizing Fasciitis (LRINEC) [16]. A total of 89 subjects with necrotizing soft tissue infections (NSTIs) were compared with 225 with severe cellulitis or abscess or both and they were stratified based on patient's complete blood count, basic metabolic panel (serum sodium, serum creatinine and serum glucose), and C-reactive protein on their admission [16]. These values were then given a score, and those patients with a score above the threshold were found to have a $92 \%$ positive predictive value and $96 \%$ negative predictive value [16]. However, due to its limitations, specifically when there are multiple inflammatory processes occurring, it should only be used when suspicion is high.

While the role of imaging in these patients may be limited, key features can appear on CT scans. Evidence of edema, fluid collection, fascial thickening and abscess formation can be helpful in directing treatment [17]. Ultrasound can also be used to detect abscess formation but lacks specificity for NF. Finally, magnetic resonance imaging (MRI) has been shown to be somewhat sensitive but falls short in specificity as tissue enhancement can be present in other conditions, such as trauma [18].

Standard treatment of NSTIs involves removal of infected or necrotic tissue via radical surgical debridement, broad 
spectrum antibiotics and resuscitation. Delay of surgical debridement is associated with increased mortality, making it the foundation of treatment. Typical debridement involves an extending of the surgical margins around the site where the NSTI is found to ensure complete removal of the necrotic tissue [1]. Most surgeries are liberal in this aspect. Follow-up and re-examination of surgical site within $24 \mathrm{~h}$ are recommended [19]. Additionally, broad spectrum antibiotics should not be delayed and must cover gram-positive, gram-negative and anaerobes. This is routinely continued until microbial culture allows de-escalation of therapy. The Infectious Disease Society of America (IDSA) current guidelines recommend vancomycin or linezolid plus one of the following therapies: piperacillintazobactam, a carbapenem, or ceftriaxone-metronidazole [19].

\section{Conclusions}

NF is a life-threatening condition with a high mortality rate if not diagnosed and treated early with antibiotics and immediate surgical intervention. This patient was actively being treated with pazopanib for recurrent RCC and presented with an insidious course of a colonic perforation, which overtime caused NF. Although gastrointestinal perforation is a rare complication associated with pazopanib, it should always be considered in patients presenting with signs of infection while on treatment. The ultimate goal of this case report is to raise awareness of the potentially rare complications associated with novel VGEFtyrosine kinase inhibitors, as well as to focus on the importance of a multidisciplinary approach to management of this rare case presentation to result in complete and full recovery.

\section{Acknowledgments}

None to declare.

\section{Financial Disclosure}

None to declare.

\section{Conflict of Interest}

None to declare.

\section{Informed Consent}

The patient described in the case report had given informed consent for the case report to be published.

\section{Author Contributions}

Each author has individually been involved in and has made substantial contributions to case selection, case presentation, discussion, drafting manuscript and revision and final approval.

\section{Data Availability}

All data and information regarding this manuscript is present within the text.

\section{References}

1. Hakkarainen TW, Kopari NM, Pham TN, Evans HL. Necrotizing soft tissue infections: review and current concepts in treatment, systems of care, and outcomes. Curr Probl Surg. 2014;51(8):344-362.

2. Stevens DL, Bryant AE. Necrotizing soft-tissue infections. N Engl J Med. 2017;377(23):2253-2265.

3. Appleby L, Morrissey S, Bellmunt J, Rosenberg J. Management of treatment-related toxicity with targeted therapies for renal cell carcinoma: evidence-based practice and best practices. Hematol Oncol Clin North Am. 2011;25(4):893-915.

4. Misiakos EP, Bagias G, Papadopoulos I, Danias N, Patapis P, Machairas N, Karatzas T, et al. Early diagnosis and surgical treatment for necrotizing fasciitis: a multicenter study. Front Surg. 2017;4:5.

5. Sarani B, Strong M, Pascual J, Schwab CW. Necrotizing fasciitis: current concepts and review of the literature. J Am Coll Surg. 2009;208(2):279-288.

6. Morua AG, Lopez JA, Garcia JD, Montelongo RM, Guerra LS. Fournier's gangrene: our experience in 5 years, bibliographic review and assessment of the Fournier's gangrene severity index. Arch Esp Urol. 2009;62(7):532540.

7. Puvanendran R, Huey JC, Pasupathy S. Necrotizing fasciitis. Can Fam Physician. 2009;55(10):981-987.

8. Wong $\mathrm{CH}$, Chang HC, Pasupathy S, Khin LW, Tan JL, Low CO. Necrotizing fasciitis: clinical presentation, microbiology, and determinants of mortality. J Bone Joint Surg Am. 2003;85(8):1454-1460.

9. Misiakos EP, Bagias G, Patapis P, Sotiropoulos D, Kanavidis $\mathrm{P}$, Machairas A. Current concepts in the management of necrotizing fasciitis. Front Surg. 2014;1:36.

10. Josko J, Gwozdz B, Jedrzejowska-Szypulka H, Hendryk $\mathrm{S}$. Vascular endothelial growth factor (VEGF) and its effect on angiogenesis. Med Sci Monit. 2000;6(5):10471052 .

11. Sloan B, Scheinfeld NS. Pazopanib, a VEGF receptor tyrosine kinase inhibitor for cancer therapy. Curr Opin Investig Drugs. 2008;9(12):1324-1335.

12. Votrient [prescribing information]. East Hanover, NJ: Novartis Pharmaceuticals Corp; 2017.

13. Ravaud A. Treatment-associated adverse event management in the advanced renal cell carcinoma patient treated with targeted therapies. Oncologist. 2011;16(Suppl 2):3244.

14. Eskens FA, Verweij J. The clinical toxicity profile of vascular endothelial growth factor (VEGF) and vascu- 
lar endothelial growth factor receptor (VEGFR) targeting angiogenesis inhibitors; a review. Eur J Cancer. 2006;42(18):3127-3139.

15. Keung EZ, Liu X, Nuzhad A, Adams C, Ashley SW, Askari R. Immunocompromised status in patients with necrotizing soft-tissue infection. JAMA Surg. 2013;148(5):419426.

16. Wong CH, Khin LW, Heng KS, Tan KC, Low CO. The LRINEC (Laboratory Risk Indicator for Necrotizing Fasciitis) score: a tool for distinguishing necrotizing fasciitis from other soft tissue infections. Crit Care Med. 2004;32(7):1535-1541.
17. Wysoki MG, Santora TA, Shah RM, Friedman AC. Necrotizing fasciitis: CT characteristics. Radiology. 1997;203(3):859-863.

18. Arslan A, Pierre-Jerome C, Borthne A. Necrotizing fasciitis: unreliable MRI findings in the preoperative diagnosis. Eur J Radiol. 2000;36(3):139-143.

19. Stevens DL, Bisno AL, Chambers HF, Dellinger EP, Goldstein EJ, Gorbach SL, Hirschmann JV, et al. Practice guidelines for the diagnosis and management of skin and soft tissue infections: 2014 update by the Infectious Diseases Society of America. Clin Infect Dis. 2014;59(2):e10-52. 